\title{
Reasons for revision surgery after orbital decompression for Graves' orbitopathy
}

\author{
Stefano Sellari-Franceschini' \\ Luca Muscatello' \\ Veronica Seccia' \\ Riccardo Lenzi' \\ Amelia Santoro' \\ Marco $\mathrm{Nardi}^{2}$ \\ Barbara Mazzi ${ }^{3}$ \\ Aldo Pinchera ${ }^{3}$ \\ Claudio Marcocci ${ }^{3}$ \\ 'Department of Neuroscience, \\ I st ENT division, ${ }^{2}$ Ophthalmology \\ Division, ${ }^{3}$ Department of \\ Endocrinology and Metabolism, \\ Orthopaedics and Traumatology, \\ Occupational Medicine, University \\ of Pisa, Italy
}

\begin{abstract}
Objectives: An analysis of complications and causes of failure in orbital decompression necessitating a second operation.

Methods: Between December 1992 and April 2007, 375 patients ( 719 orbits) were operated on using various techniques. Fourteen patients were initially operated on in our unit: 8 (group A1) were re-operated on after a short time due to complications connected with the decompression operation, 7 (group A2) were operated on after some time due to recurrence of the illness or unsatisfactory decompression (one patient is in both group A1 and A2). Five patients (group B) underwent a first operation elsewhere.
\end{abstract}

Results: For group A1 the most serious complications were connected to the nasal approach. For group A2 the operations were performed either because of a neuropathy recurrence or for further proptosis reduction due to recurrence or patient dissatisfaction. Lack of preoperative data hinders conclusions about group B, apart from one patient where the operation had not resolved a serious optic neuropathy after decompression based on Olivari technique combined with three-wall operation according to Mourits and colleagues (1990).

Conclusions: We can deduce from group A1 that extreme attention is necessary during endonasal access, from group A2 that balancing the eyes is advisable, sacrificing maximum proptosis reduction to gain greater patient satisfaction, and from group B that decompression of the orbital apex is fundamental in the case of neuropathy.

Keywords: orbital decompression, Graves' orbitopathy, revision surgery

\section{Orbital decompression}

The clinical manifestations of Graves' orbitopathy (GO) can be variable and patients may require surgery for cosmetic problems, severe proptosis or optic neuropathy. Several surgical techniques have been adopted, including the removal of retro-orbital fat (Olivari 1991), one or more orbital walls (Walsh and Ogura 1957; Maroon and Kennerdell 1982; Hurwitz and Birt 1985; Kennedy et al 1990; Mourits et al 1990; Garrity et al 1993; Rootman et al 1995; Goldberg et al 2000; Graham et al 2003), or both together. For this reason, and because of the small number of patients participating in each study, it is difficult to evaluate the results and to understand the advantages and the risks of one surgical approach compared with another. Surgical complications are rarely described and no information is given as to whether these patients required further operations.

We herein report the results of a retrospective study on 19 patients, 14 operated at our unit, and 5 elsewhere, who required a second operation, and analyze the reasons of further surgery.

\section{Materials and methods}

We reviewed the medical records of 375 patients with GO (719 orbits) operated on at 1st ENT unit, University of Pisa, between December 1992 and April 2007. 
Table I Surgical techniques used from December 1992 to April 2007

\begin{tabular}{lll}
\hline Technique & No of patients & No of orbits \\
\hline Walsh-Ogura & 20 & 37 \\
Mourits et al & 21 & 37 \\
Walsh-Ogura and Mourits et al & 31 & 56 \\
Medial and/or lateral approach & 303 & 589 \\
Total & $\mathbf{3 7 5}$ & $\mathbf{7 1 9}$ \\
\hline
\end{tabular}

The patients were operated on using various techniques, including the Walsh-Ogura (WO) (Walsh and Ogura 1957), the 3 walls decompression according to Mourits and colleagues (M) (Mourits et al 1990), and a balanced decompression with medial and lateral wall approach (Graham et al 2003; Sellari-Franceschini et al 2005) (Table 1). Fourteen patients required a second orbital surgery. All but one had previously been operated using the balanced decompression technique. One patient was operated using both the WO and the M technique. Eight patients (Group A1) required a second operation due to a complication closely connected to the first operation (Table 2) and seven (group A2) needed further decompression because of residual proptosis (Table 3 and 4). One patient (1) is in both group A1 and A2.

We also studied five patients (group B) who underwent orbital decompression elsewhere using the Olivari's technique (O) (Olivari 1991). Four of them required further surgery because of insufficient results and one because of persistent severe sight loss (Table 5).

\section{Results \\ Group A I}

Four patients were operated on again due to complications connected with the endonasal approach. One patient (3) required re-operation because of bleeding upon removal of the nasal swabs on the second day after the operation, while another (7) was operated on again three years later for a mucocele in the posterior part of the ethmoid, which was causing proptosis. The two remaining patients had more serious complications. Patient 1, after the nasal swabs had been removed on the second day, started complaining of strong headache after blowing the nose. A computed tomography (CT) scan showed a pneumocephalus associated to cerebrospinal fluid (CSF) leak (Figure 1). The fistula was surgically closed and the pneumocephalus resolved in ten days without sequelae. One patient (8) complained on the second day after decompression of monolateral visual loss. A CT scan showed a fragment of papiracea pushing against the medial rectus muscle, with compression of the optic nerve (Figure 2). After removing the fragment the visual function steadily recovered until normal.

Of the remaining 4 patients, one (2) complained of reduction in the visual field monolaterally nine days after the operation. A CT scan was performed and showed

Table 2 Group A I: patients who have undergone a second operation due to a complication closely connected to the first operation

\begin{tabular}{|c|c|c|c|}
\hline Patient (sex) & Surgical approach & Complication & Treatment \\
\hline I (f) & $L+M$ & 2nd postoperative day: CSF leak and pneumocephalus & Surgical repair of the CSF leak \\
\hline $2(f)$ & $L+M$ & 9th postoperative day: left optic nerve compression & $\begin{array}{l}\text { Transnasal removal of a bone fragment from the } \\
\text { left orbit }\end{array}$ \\
\hline $3(\mathrm{~m})$ & $L+M$ & $\begin{array}{l}\text { 2nd postoperative day: anterior and posterior nasal } \\
\text { bleeding }\end{array}$ & Right sphenopalatine artery cauterization \\
\hline $4(\mathrm{~m})$ & L & $\begin{array}{l}\text { Few hours after the operation: left orbital } \\
\text { hemorrhage }\end{array}$ & Hemostasis \\
\hline $5(\mathrm{~m})$ & $L+M$ & $\begin{array}{l}\text { 2nd postoperative day: bleeding from the left } \\
\text { temporalis muscle }\end{array}$ & Hemostasis \\
\hline $6(\mathrm{~m})$ & $L+M$ & $\begin{array}{l}\text { Ist postoperative day: patient's self-removal of the } \\
\text { right orbital drainage and consequent blood collecting } \\
\text { in the orbit }\end{array}$ & Hemostasis and new drainage application \\
\hline $7(\mathrm{~m})$ & $L+M$ & $\begin{array}{l}3 \text { years after the operation: mucocele of the left } \\
\text { posterior ethmoidal cells }\end{array}$ & Marsupialization of the mucocele \\
\hline $8(f)$ & $L+M$ & 2nd postoperative day: right optic nerve compression & $\begin{array}{l}\text { Transnasal removal of a bone fragment from the } \\
\text { right orbit }\end{array}$ \\
\hline
\end{tabular}

Abbreviations: CSF, cerebrospinal fluid; L, transpalpebral lateral orbital wall removal; M, endonasal medial orbital wall removal. 
Table 3 Group A2: pre- and post-operative values of proptosis in patients undergoing the first operation

\begin{tabular}{|c|c|c|c|c|c|}
\hline \multirow[t]{2}{*}{ Patient (sex) } & \multicolumn{2}{|c|}{ Preoperative proptosis } & \multirow[t]{2}{*}{ Technique } & \multicolumn{2}{|c|}{ Postoperative proptosis } \\
\hline & Right eye & Left eye & & Right eye & Left eye \\
\hline$I(f)$ & 29 & 27 & $\mathrm{WO}+\mathrm{M}$ & 23 & 21 \\
\hline $9(\mathrm{f})$ & 26 & 26 & Medial plus lateral wall removal & 24 & 23 \\
\hline $10(f)$ & 22 & 21 & Medial plus lateral wall removal & 16,5 & 15,5 \\
\hline II (m) & 26 & 27 & Medial plus lateral wall removal & 22 & 22 \\
\hline $12(\mathrm{~m})$ & 25 & 24,5 & Medial wall removal & 23 & 21 \\
\hline $13(f)$ & 25 & 23 & Medial plus lateral wall removal & 21,5 & 19 \\
\hline $14(f)$ & 22 & 24 & medial plus lateral wall removal & 17 & 19 \\
\hline
\end{tabular}

Abbreviations: WO,Walsh-Ogura; M, Mourits et al.

the presence of a bone fragment from the sphenoid wall which was pushing against the optic nerve (Figure 3). The bone fragment was surgically removed with a slow steady recovery of visual field. Three patients $(4,5$, and 6) were operated on the second and third day after surgery because of sudden and rapid onset of post-operative monolateral proptosis with ipsilateral chemosis (Figure 4). A CT scan was performed and in all cases blood collection in the lateral area of the orbit was detected and removed surgically.

\section{Group A2}

Two patients (9 and 11) were operated on owing to insufficient bilateral decompression. One patient (1) had worsening of proptosis because of a recurrence of GO after seven years, and another (10) was re-operated on twice due to recurrence of GO with a slight reduction in the visual field, first on the left eye and then on the right eye, one and three years after the first operation, respectively (Figure 5). A transnasal approach with removal of small fragments of residual papiracea and posterior orbital floor was performed.
In both patients the operations were successful, with complete recovery of vision.

Patients 13 and 14 were operated on monolaterally, due to persistent asymmetry in the two eyes. Both patients underwent a nasal approach with removal of the residual lamina papiracea, where possible, removal of the middle turbinate and partial removal of the floor, the greater sphenoidal wing having been already completely removed (Figure 6).

Patient 12 refused the external approach due to fear of ugly eyelid scars and was submitted to a transnasal approach, operating only on the medial wall. Because of insufficient reduction in proptosis, he then accepted a transeyelid approach to the lateral wall. The results obtained are shown in Tables 3 and 4.

\section{Group B}

As far as group B is concerned, it is difficult to ascertain the reasons of the first failure in the 4 patients who required further decompression for persistent proptosis, since information regarding the preoperative status were scarce.

Table 4 Group A2: pre- and postoperative values of proptosis in patients undergoing the revision surgery

\begin{tabular}{|c|c|c|c|c|c|}
\hline \multirow[t]{2}{*}{ Patient (sex) } & \multicolumn{2}{|c|}{ Preoperative proptosis } & \multirow[t]{2}{*}{ Technique } & \multicolumn{2}{|c|}{ Postoperative proptosis } \\
\hline & Right eye & Left eye & & Right eye & Left eye \\
\hline I (f) & 23 & 21 & Medial plus lateral wall removal & 20 & 20 \\
\hline $9(f)$ & 24 & 23 & Medial wall removal & 23 & 22 \\
\hline $10(f)$ & 15.5 & 16.5 & Medial wall removal & 15.5 & 14 \\
\hline II (m) & 22.5 & 22.5 & Removal of the posterior part of the floor & 21.5 & 21.5 \\
\hline $12(\mathrm{~m})$ & 23 & 21 & $\begin{array}{l}\text { Right medial plus lateral wall removal and } \\
\text { left lateral wall removal }\end{array}$ & 16.5 & 16 \\
\hline $13(f)$ & 21.5 & - & Right medial wall removal & 21 & - \\
\hline $14(f)$ & - & 19 & Left medial wall removal & - & 19 \\
\hline
\end{tabular}


Table 5 Group B: patients who had already undergone an operation somewhere else and had been referred to us for revision

\begin{tabular}{llll}
\hline Patient (sex) & $\begin{array}{l}\text { Technique } \\
\text { (Ist operation) }\end{array}$ & $\begin{array}{l}\text { Technique } \\
\text { (2nd operation) }\end{array}$ & $\begin{array}{l}\text { Reason for } \\
\text { revison surgery }\end{array}$ \\
\hline $15(\mathrm{~m})$ & O + M & Bilateral medial and lateral wall removal & ON \\
$16(\mathrm{~m})$ & O and K & Bilateral medial and lateral wall removal & RP \\
$17(\mathrm{f})$ & $\mathrm{O}$ & Bilateral medial and lateral wall removal & RP \\
$18(\mathrm{f})$ & $\mathrm{O}$ & Bilateral medial and lateral wall removal & RP \\
$19(\mathrm{f})$ & $\mathrm{O}$ & Left medial and lateral wall removal & RP \\
\hline
\end{tabular}

Abbreviations: O, Olivari technique; M, Mourits et al; K, Kennedy et al; ON, optic neuropathy; RP, residual proptosis.

The remaining patient (15) has been already described in a previous paper (Sellari-Franceschini et al 2005). He was re-operated because of a serious optic neuropathy that persisted after the previous operation, which was performed using the $\mathrm{O}$ technique associated with a coronal decompression a la $\mathrm{M}$. The CT scan showed the persistence of all bone structures at the apex of the orbit and compression on the optic nerve by the extraocular muscles. A new transnasal approach was performed, removing the posterior part of the lamina papiracea and the posterior part of orbital floor; in addition the great sphenoidal wing was completely removed through a trans-eyelid approach. The recovery of vision was rapid and significant and the

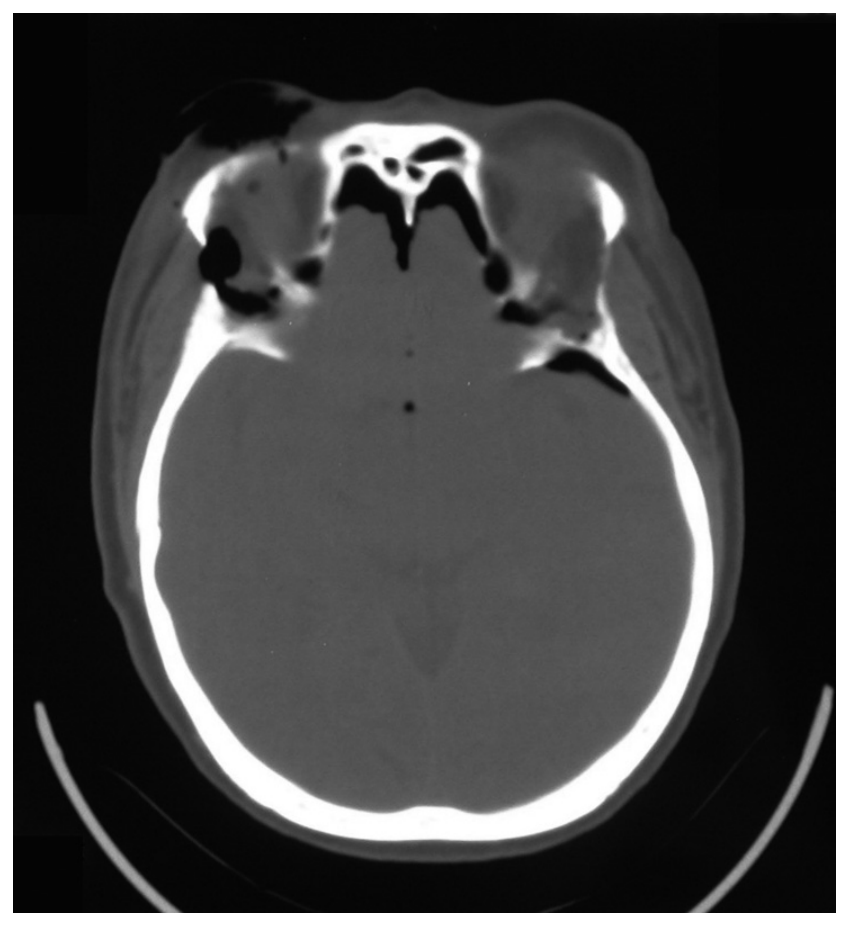

Figure I Postoperative CT scan showing the presence of a pneumocephalus. computerized analysis of the visual field showed an almost complete recovery.

\section{Discussion}

Several surgical approaches have been described for orbital decompression in GO and the choice in each medical center often depends on the local surgeon expertise. There are only few surgeons who perform different approaches based on individual needs and have a follow-up of large series of patients. Therefore, it is difficult to understand from the literature which complications are associated with a given surgical approach, and how often a second surgery is needed for their solution.

\section{Group A I}

Our experience indicates that three patients of group A1 needed to be re-operated shortly after the first surgery because of hemorrages in the outer region of the orbit, due to bleeding from the orbital fat and temporalis muscle. One patient accidentally removed the drainage by himself immediately after the operation instead of having it left in place for 24 hours, and two other patients bled within the first day after removal of the drainage. Thus the observed rate of bleeding ( 3 out of 719 orbit) is extremely low and therefore it is fair to conclude that a 24-hour drainage period is usually sufficient.

The complications associated with the endonasal approach were more serious. The epistaxis appearing when the swabs were removed required a simple hemostasis procedure. Of the two patients ( 2 and 8 ) with compression of the optic nerve by bone fragments, the one with an indirect compression via the medial rectus muscle and operated on the third day had a complete recovery in few weeks, whilst the other with a direct compression on the nerve and the surgery performed with a delay of ten days recovered completely in two years. CSF leak was also associated with a serious complication, ie, pneumocephalus, which in any case cleared without any residual effect. 

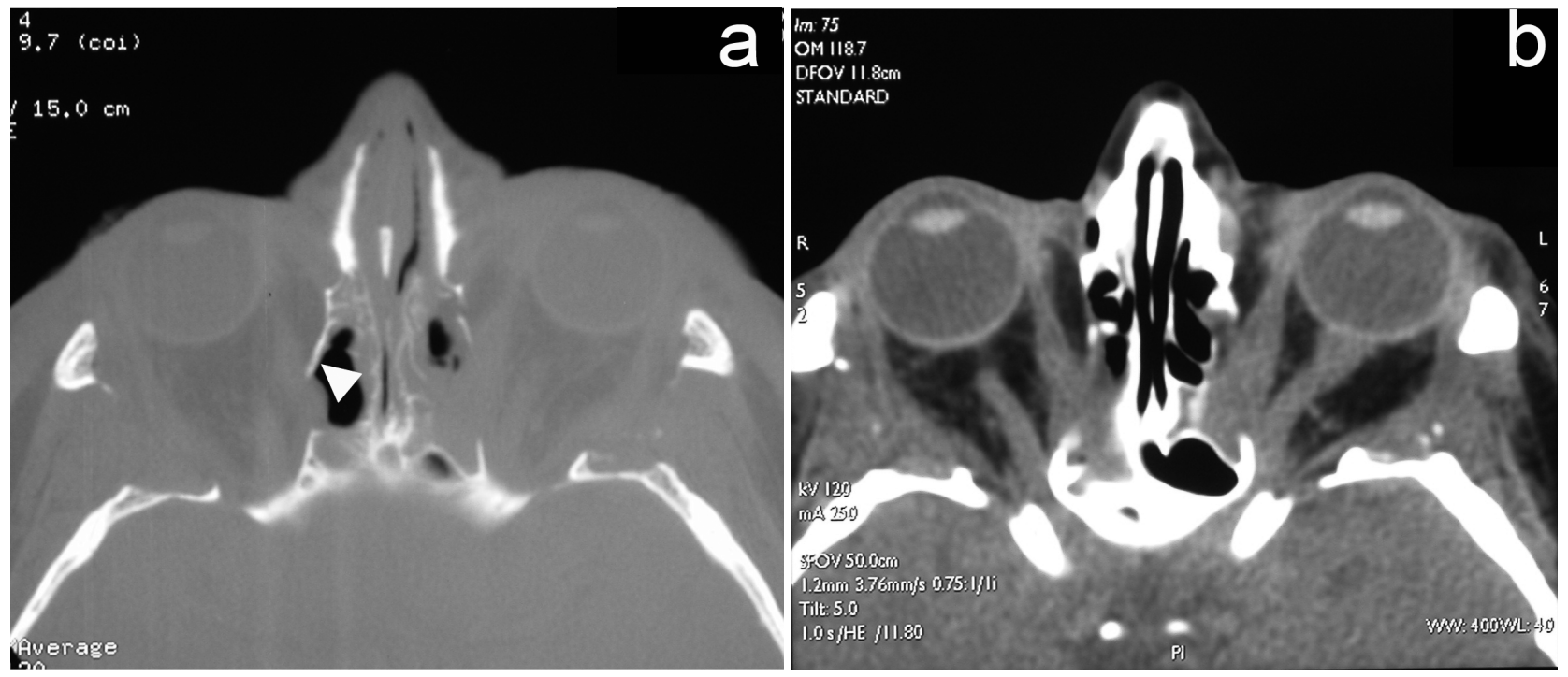

Figure $\mathbf{2}$ CT scan showing the fragment of papiracea (white arrow) pushing against the right medial rectus muscle (a); the fragment was then removed with a new transnasal surgical procedure (b).

\section{Group A2}

As far as group A2 is concerned, two patients had to be operated on again due to recurrence of GO. The first (1) was operated on bilaterally, seven years after the first operation. The other one (10) required two additional surgical procedures because of newly developed slight loss in the left eye after one year and in the right eye after three years. A transnasal approach with removal of small fragments of residual papiracea and posterior orbital floor was sufficient to rectify the situation. At the time of both revision surgeries, the patient was euthyroid on L-T4 replacement therapy and TRAb were negative. In our experience, that was the only case of two consecutive recurrences of disease with optic neuropathy after orbital decompression.

Two patients (9 and 11) were operated on bilaterally due to insufficient decompression after the first operation. In these patients the emphasis on trying to avoid a postoperative diplopia brought about a limited medial approach and the result of the decompression was not satisfactory.

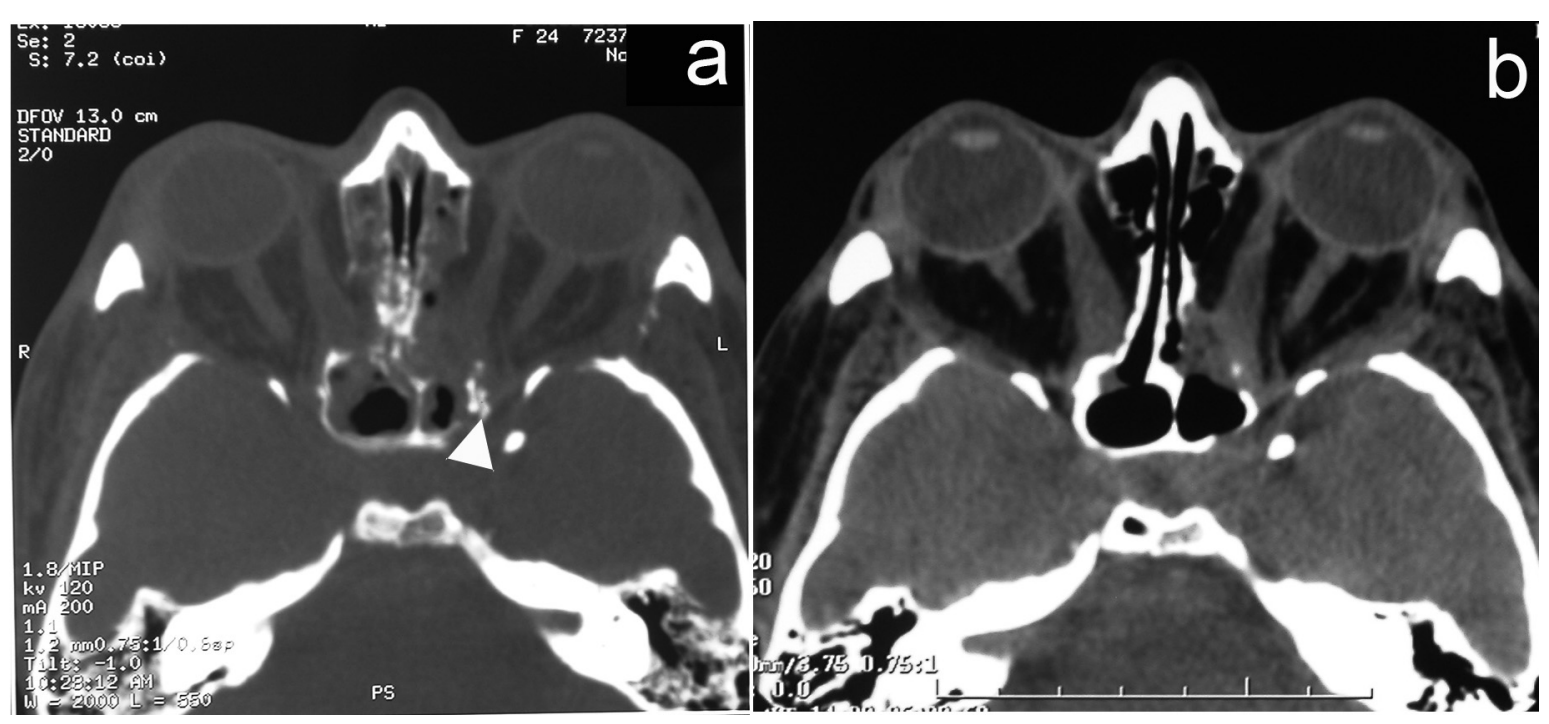

Figure 3 CT scan showing a bone fragment from the sphenoid (white arrow) compressing the left optic nerve (a); after the transnasal removal of the bone fragment, a new CT scan showed the successfully decompressed optic canal (b). 


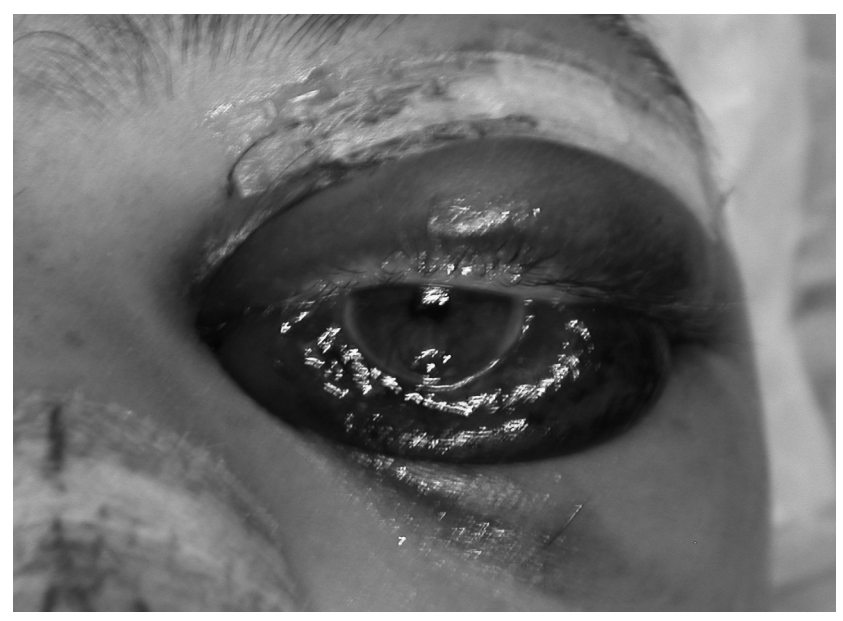

Figure 4 Left eye of a patient undergoing a second operation for orbital hemorrage.

Two patients (13 and 14) were operated on again monolaterally because of eye asymmetry after the decompression. Very often the patients have a different proptosis in the two eyes and, when the difference is greater than $2 \mathrm{~mm}$, there is the risk that the asymmetry will persist after decompression. From the patient's perspective, this result is felt much more as a failure than a globally lesser, but balanced, reduction in proptosis. In these two patients the second operation, in which particular attention was paid to avoid diplopia in primary gaze, turned out to be only partially satisfactory for the patients, because of a residual minor imbalance and development of diplopia in the lateral gaze.

The patient (18) who refused a combined approach subsequently accepted a second operation with an external approach. He was fully satisfied with the result.

\section{Group B}

As far as patients of group B are concerned, four were initially operated on using the $\mathrm{O}$ technique and one patient was operated endoscopically with the 2 walls removal technique according to Kennedy and colleagues (1990). It is difficult to comment on these patients due to the lack of accurate information on the preoperative conditions.

The patient (15) with persistent optic neuropathy was initially operated on using the $\mathrm{O}$ technique in combination with bone decompression through a coronal approach a la $\mathrm{M}$. The CT scan showed that the limited opening of the lateral and medial walls did not resolve the compression on the optic nerve at the orbital apex, suggesting that in patients with optic neuropathy it is necessary to perform a wide opening of the bone walls at the orbital apex.

\section{Conclusions}

Because of the small series of patients and the lack of homogeneity our study does not allow to drawn definite conclusions, but some considerations can be made. In patients with optic neuropathy it is very important to obtain an adequate decompression at the orbital apex. In one of our patients, the $\mathrm{O}$ technique, even if associated to a partial opening of the orbital walls, was not adequate to provide satisfactory results. Indeed, a wide opening of the bone at the orbital apex should be advisable.

As far as the complications closely tied to the decompression procedure are concerned, those associated with the endonasal approach were more serious.

We experienced two cases of optic nerve compression due to bone fragments. In one case surgical manipulation of the lateral wall of the sphenoid sinus led to the formation of a bone fragment compressing the optic nerve in the optic canal. In the other case a fragment of lamina papiracea provoked the compression of the medial rectus muscle and then of the optic nerve. Here, CT findings didn't correspond to the symptoms, but the surgical removal of the fragment solved the visual loss.

Such complications are not reported in the literature, where also complications in general are fairly mentioned.

We are dealing with two cases on 719 orbits decompressed. Therefore it's a rare complication that always required a surgical management.

Thus, extreme attention is needed in performing the ethmoidectomy and opening of the papiracea, particularly in the posterior section.

Hemorrhages in the lateral part of the orbit were rather rare and easy to control.

Concerning the operations performed for persistent proptosis, we have noticed that, even if it was considerably reduced by first surgery, a difference in proptosis between the two eyes was felt by the patient as a failure. A lesser, yet balanced, reduction in proptosis was accepted more willingly. Since the two eyes are often involved in the disease with different severity, balancing the decompression is easier when operating simultaneously on the two eyes. In our experience is often more difficult to obtain a good decompression in the eye with a higher preoperative proptosis. Therefore, in cases where the preoperative difference in proptosis between the two eyes is greater than 2 $\mathrm{mm}$, the surgeon should address firstly the worst eye and then adapt the extent of decompression in the other eye in order to obtain a symmetric reduction in proptosis. 

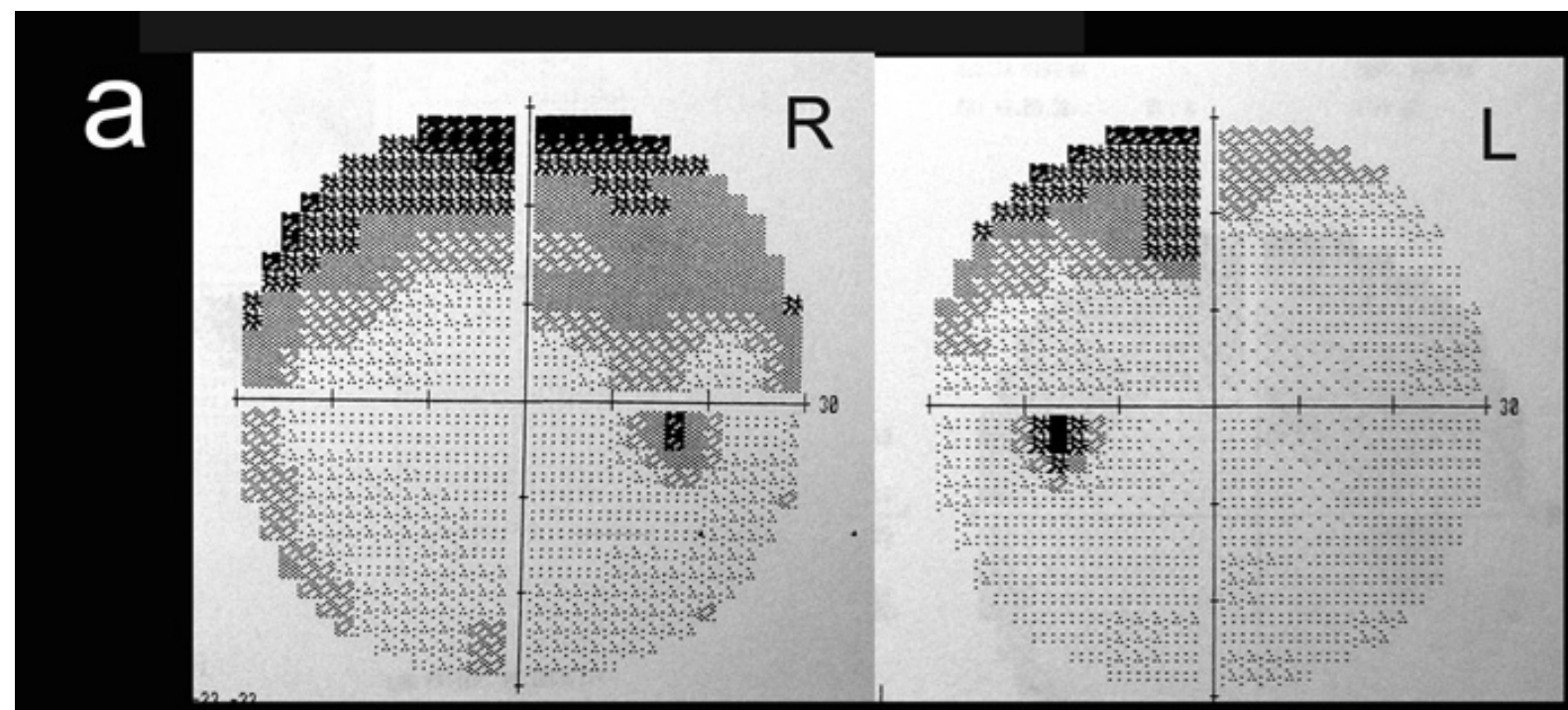

b
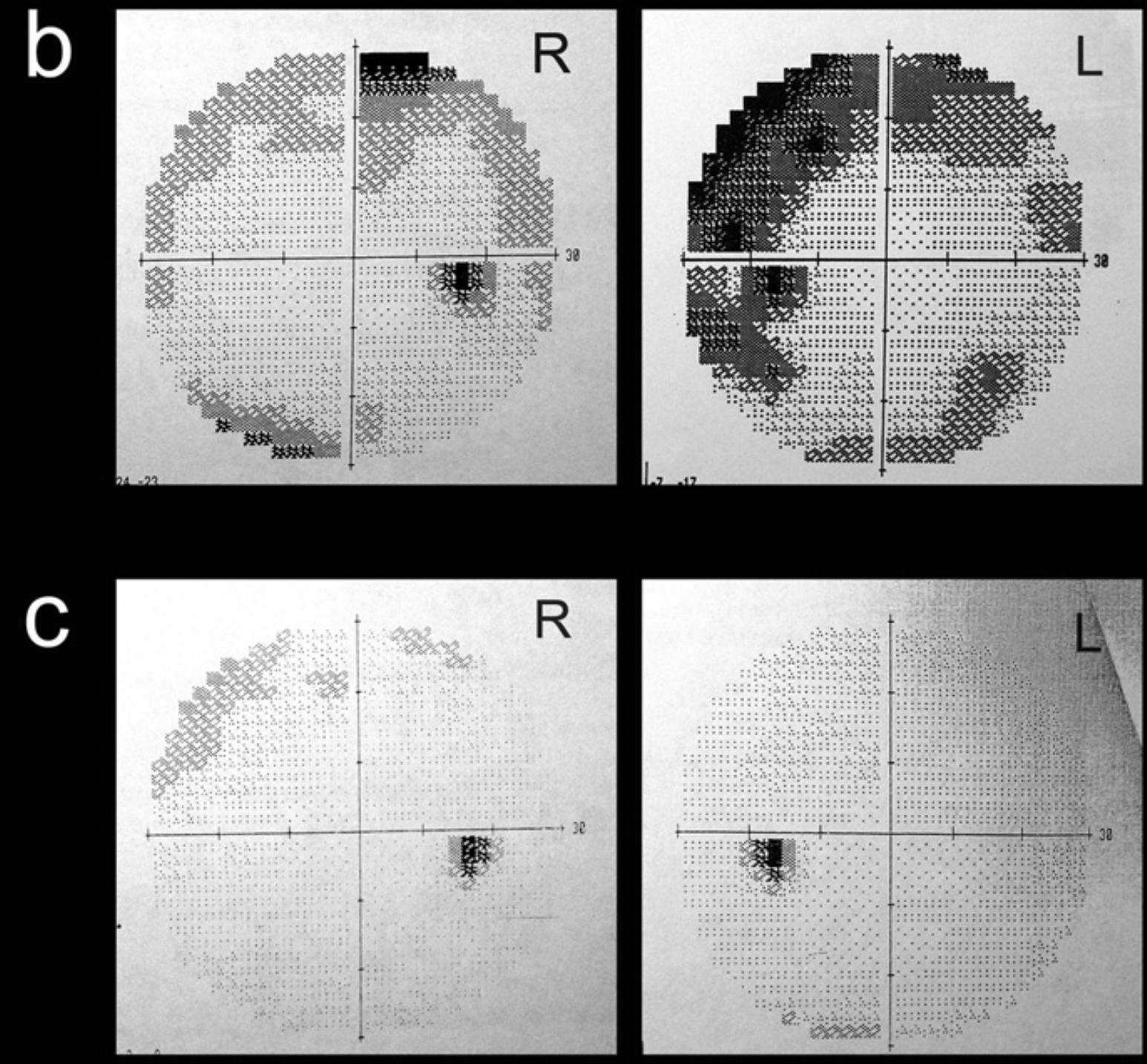

Figure $\mathbf{5}$ Visual fields of a patient operated on both eyes for recurrence of GO made before the first operation (a), before revision surgery (b) and after revision surgery (c). 


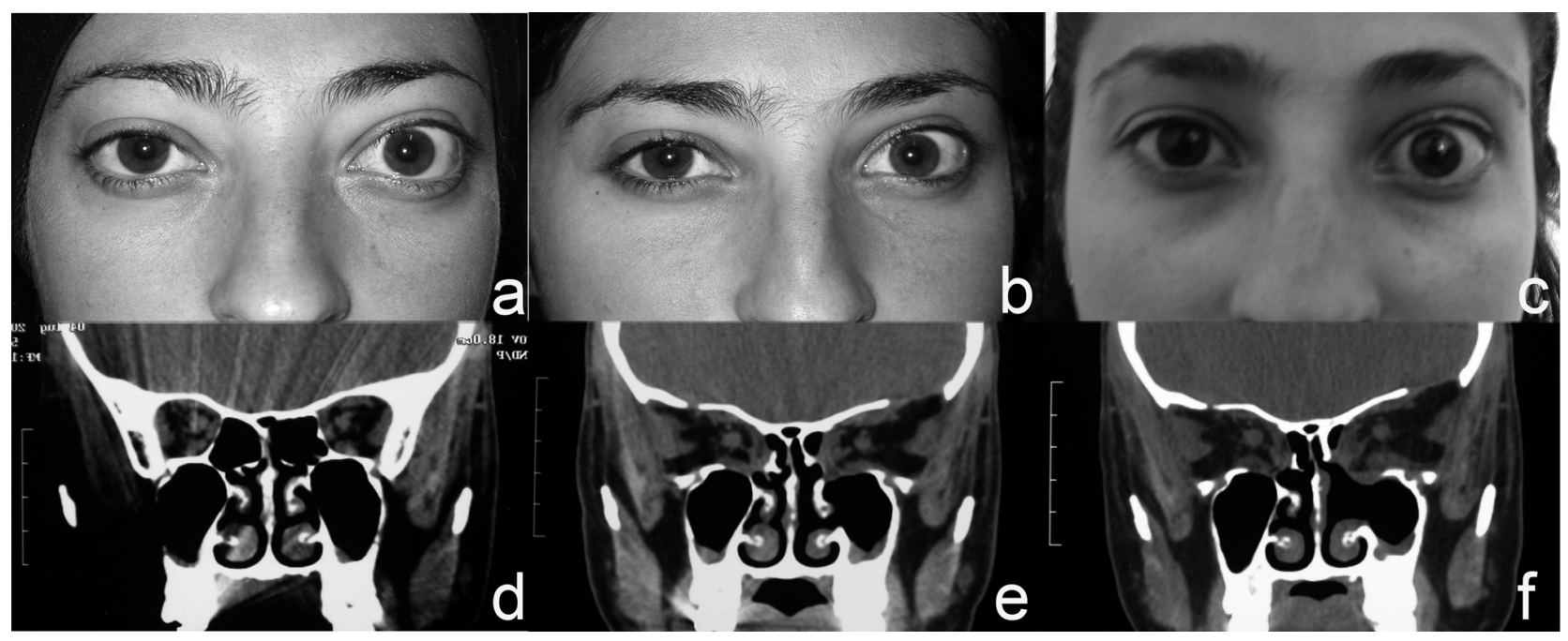

Figure 6 Photographs and CT scans of patient number 14 before $(\mathbf{a}, \mathbf{d})$, between $(\mathbf{b}, \mathbf{e})$ and after $(\mathbf{c}, \mathbf{f})$ the two operations.

\section{References}

Garrity JA, Fatourechi V, Bergstralh EJ, et al. 1993. Results of transantral orbital decompression in 428 patients with severe Graves' ophthalmopathy. Am J Ophthalmol, 116:533-47.

Goldberg RA, Perry JD, Hortaleza V, et al. 2000. Strabismus after balanced medial plus lateral wall versus lateral wall only orbital decompression for dysthyroid orbitopathy. Ophthal Plast Reconstr Surg, 16:271-7.

Graham SM, Brown CL, Carter KD, et al. 2003. Medial and lateral orbital wall surgery for balanced decompression in thyroid eye disease. Laryngoscope, 113:1206-9.

Hurwitz JJ, Birt D. 1985. An individualized approach to orbital decompression in Graves' orbitopathy. Arch Ophthalmol, 104:660-5.

Kennedy DW, Goodstein ML, Miller NR, et al. 1990. Endoscopic transnasal orbital decompression. Arch Otolaryngol Head Neck Surg, $116: 275-82$.
Maroon JC, Kennerdell JS. 1982. Radical orbital decompression for severe dysthyroid exophthalmos. J Neurosurg, 56:260-6.

Mourits PhM, Koornneef L, Wiersinga WM, et al. 1990. Orbital decompression for Graves' ophthalmopathy by inferomedial, by inferomedial plus lateral, and by coronal approach. Ophthalmology, 97:637-41.

Olivari N. 1991. Transpalpebral decompression of endocrine ophthalmopathy (Graves' disease) by removal of intraorbital fat: experience with 147 operations over 5 years. Plast Reconstr Surg, 87:627-43.

Rootman J, Stewart B, Goldberg RA. 1995. Orbital surgery: a conceptual approach, 1st ed. Philadelphia: Lippincott-Raven Publisher.

Sellari-Franceschini S, Berrettini S, Santoro A, et al. 2005. Orbital decompression in Graves' ophthalmopathy by medial and lateral wall removal. Otolaryngol Head Neck Surg, 133:185-9.

Walsh TE, Ogura JH. 1957. Transantral orbital decompression for malignant exophthalmos. Laryngoscope, 67:544-9. 